\title{
Determining the Relationship among Organizational Commitment, Occupational Stress, and Interpersonal Relations according to Adult Attachment Styles of Clinical Dental Hygienists
}

\author{
Mi-Hyun Choi, ${ }^{1, \dagger}$ and Hee-Hong $\mathrm{Min}^{2}$ \\ ${ }^{1}$ Kim Yang-jung Dental Office, Daejeon 35271, \\ ${ }^{2}$ Department of Dental Hygiene, Daejeon Health Institute of Technology, Daejeon 34504, Korea
}

\begin{abstract}
Background: This study aimed to identify the types of adult attachment and determine the relationship between adult attachment and job factors in dental hygienists. Ultimately, it was necessary to identify the need for a secure attachment to improve the quality of clinical dental hygienist's services.

Methods: Data of 454 clinical dental hygienists working in dental hospitals or clinics were collected. The research tools consisted of items related to the general and work characteristics of dental hygienists ( 9 items), adult attachment styles (36 items), organizational commitments (12 items), occupational stress (15 items), and interpersonal relations (18 items). Cronbach's $\alpha$ of each tool was $\geq 0.7$.

Results: Most of the participants had fearful attachment styles, followed by dismissing-avoidance, security, and preoccupation. Security was the highest level of organizational commitment according to the adult attachment style, although the differences of the levels were insignificant. For occupational stress, preoccupation was the highest, followed by fearful, security, and dismissing-avoidance, and the differences were significant $(p<0.001)$. For interpersonal relations, security was the highest, followed by preoccupation, dismissing-avoidance, and fearful in order, and the differences were significant $(\mathrm{p}<0.001)$.

Conclusion: Job stress and interpersonal relation ability according to the adult attachment style of clinical dental hygienists had significant results. Thus, the development of attachment improvement programs by personal style, development of differentiated clinical education and its application, and improvements in the adult attachment styles of clinical dental hygienists would be required rather than simply presenting the needs to collectively improvement the working environment.
\end{abstract}

Key Words: Adult attachment styles, Dental hygienist, Interpersonal relations, Occupational stress, Organizational commitment

\section{Introduction}

Today, having a good social life takes an enormous mental energy. Although most people could be in a similar situation, they tend to respond differently. Attachment, an important role in social development, is developed during infancy. Secure attachment is developed in a safety-based attachment relationship, and the internal working model created at this time is maintained and continuously influences the development of relationships with others after infancy $^{1)}$.

Among adult attachment studies, Bartholomew and

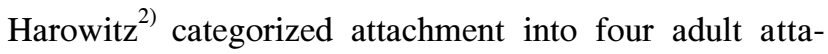
chment styles: secure, preoccupied, dismissing, and fearful ${ }^{1}$. According to these studies, those with a secure attachment style were capable of developing intimate relationships with others, while those with a fearful attachment style were afraid to engage in intimate relationships and struggled to develop intimate relationships with others. Brennan et al. ${ }^{3)}$ collected the 
existing self-report measures of attachment and analyzed the factors; they classified these factors into secure, preoccupied, dismissing, and fearful attachments based on the two large dimensions: avoidance and anxiety ${ }^{1)}$.

If an individual has a limited attachment to his or her parents during childhood, the attachment that is formed after the individual becomes an adult will continue to extensively affect his or her relationships with others and will have a significant impact on work and personal growth. Additionally, adult attachment can play various roles; it can help form an attachment between individuals, develop companionship, or allow individuals to share experiences ${ }^{1)}$. The adult attachment provides a secure base from which an individual can explore their daily activities; thus, individuals can work, take a rest, and play while forming secure attachment with others ${ }^{4)}$.

In previous studies related to adult attachment styles, individuals who developed a secure adult attachment showed the highest self-confidence than those with an insecure attachment, and they pursued social support or used problem-focused coping style when stressed ${ }^{5)}$. Anger management, depression, and professional self-concept varied according to attachment styles, and professional self-concept was higher among individuals who developed secure attachment styles than among those who developed insecure attachment styles ${ }^{6}$.

Studies on other occupations and duties demonstrated that those with secure style showed high organizational commitment and job satisfaction with low occupational stress, which were desirable outcomes, but those with insecure style showed lower organizational commitment and job satisfaction than other styles with highest level of work-related stress ${ }^{4)}$. Individuals with secure and preoccupied attachments had higher scores than those with dismissing and fearful attachments on interpersonal relations $^{7)}$. Thus, adult attachment styles are important emotional factors for living and have a significant impact on interpersonal relations and job experiences. In other words, in adult attachment styles, adults can form a secure attachment to trust others and develop positive interpersonal relations $^{1)}$. A dental hygienist is a specialist in oral disease prevention and health promotion and who have a strong work ethic. A secure attachment is an important factor for dental hygienists in order to help people they meet maintain optimal oral health.

Based on the results of work-related studies, dental hygienists were more likely to experience work-related stress as their work burden, time pressure, and job position increase $^{8)}$, and their personality types also affected stress ${ }^{9,10)}$. Furthermore, emotional organizational commitment was affected by interpersonal relations and social benefits ${ }^{11)}$. Most dental hygienists deal with high levels of stress and are exposed to higher risks of social psychological stress $^{12)}$. Previous studies suggested that the behavioral patterns (dominance, influence, steadiness, and conscientiousness) similar to adult attachment styles had a great impact on the levels of work-related stress ${ }^{13)}$. Job-related factors, such as organizational commitment or workrelated stress of dental hygienists, will highly likely to affect their work. Each attachment style is considered to have different acceptance levels in the occupational environment as the hygienist performs his or her duties. It can influence job satisfaction and organizational satisfaction and affect relationships with colleagues and patients under their care.

For such reasons, research on adult attachment of dental hygienists is considered to be necessary; however, no previous study has been conducted to evaluate this phenomenon. Therefore, this study aimed to investigate the current adult attachment styles of dental hygienists and determine the relationships between job-related factors and adult attachment. Ultimately, the present study aimed to identify the necessity of promoting secure attachments to improve the quality of clinical dental hygienists' services.

\section{Materials and Methods}

\section{Study participants}

Clinical dental hygienists who worked in dental hospitals or dental clinics located in Seoul, Gyeonggi-do, Chungcheong-do, Jeolla-do, and Gyeongsang-do were selected as study participants using convenience sampling from September 1, 2018 to October 23, 2018. The sample size was calculated using $G^{*}$ Power version 3.1.9 program; assuming a medium effect size of 0.15 , a significance level of 0.05 , a power of 0.95 , and 6 predictor variables (adult 
attachment styles, organization commitment, work-related stress, interpersonal relations, general characteristics, and job characteristics) required for one-way ANOVA, the minimum number of samples was 429, and 463 participants were selected considering dropouts. We made an online questionnaire to conduct the study and explained the purpose of the survey. The participants were assured that their answers will remain confidential and used only for research purposes. Of the total responders, the questionnaires of 454 participants $(98.1 \%)$ were used in the final analysis, and those of 9 participants (1.9\%) with missing or insufficient data were excluded.

\section{Study methods}

\section{1) General characteristics and job characteristics}

The questionnaires consisted of five questions about general characteristics (age, marriage, education, workplace, and work experience) and four questions related to job position, work relationship, experience of conflict in workplace, and considerations for a career change.

\section{2) Adult attachment styles}

Adult attachment of dental hygienist was assessed using the experiences in close eelationships scale, a self-report measurement of adult attachment developed by Brennan et al. ${ }^{3)}$, which was translated by Hwang ${ }^{14)}$ and has been used in the studies conducted by $\mathrm{Oh}$ and Sung ${ }^{4)}$, targeting nurses. In this instrument, the items were rated using a five-point Likert scale ranging from 1="strongly disagree" to $5=$ "strongly agree." Items 1 and 6 with factor loadings of $<0.4$ were removed as the validity test showed a cumulative covariance of 58.09. The questionnaire had a total of 36 items: 18 items were related to the avoidance dimension and the other 18 were related to the anxiety dimension. Higher scores denote higher levels of anxiety and lower levels of adult attachment. Based on the mean scores of participants in the avoidance and anxiety dimensions of the adult attachment styles, those who obtained lower scores in the avoidance and anxiety dimensions were classified as having secure attachments, those who had high scores in the avoidance dimension but lower scores in the anxiety dimension were classified as having dismissing attachments, those who had lower scores in the avoidance dimension but higher scores in the anxiety dimension were classified as having preoccupied attachments, and those who had higher scores in the avoidance and anxiety dimensions were classified as having fearful attachments. In a study by Brennan et al. ${ }^{3)}$, Cronbach's $\alpha$ for the avoidance and anxiety dimensions were 0.94 and 0.91 , respectively; in contrast, Cronbach's $\alpha$ for the avoidance and anxiety dimensions in the present study were 0.775 and 0.857 , respectively.

\section{3) Organizational commitment}

To assess the level of organizational commitment of dental hygienists, an instrument developed by Allen and Meyer ${ }^{15)}$ and used by $\mathrm{Oh}^{16)}$ was utilized. This instrument uses a five-point Likert scale ranging from 1="strongly disagree" to $5=$ "strongly agree," and negatively phrased items were re-coded in the opposite direction. The original study instrument was used since the validity test showed a cumulative covariance of $68.66 \%$ and a factor loading of $\geq 0.4$. A higher score indicates that the degree of organizational commitment is high. This instrument was consist of 12 items. In a study by $\mathrm{Oh}^{16)}$, Cronbach's $\alpha$ was 0.853 , and that in the present study was 0.923 .

\section{4) Occupational stress}

To assess the levels of occupational stress of dental hygienists, an instrument, which was developed by Kim and $\mathrm{Gu}^{17)}$ to measure the levels of stress that nurses had experienced while working, was modified and supplemented to fit to dental hygienists. The instrument was used after removing items $1,2,7,11,12$, and 14 with a factor loading of $<0.4$ and a cumulative covariance of $57.65 \%$. In this nine-item instrument, items were rated using a five-point Likert scale. A higher score indicates higher stress levels. In a study by Kim and $\mathrm{Gu}^{17)}$, Cronbach's $\alpha$ was 0.956 , while that in this study was 0.842 .

\section{5) Interpersonal relations}

To assess one's interpersonal skills, the relationship change scale, which was developed by Guerney ${ }^{18)}$, translated by Moon ${ }^{19)}$, and modified and supplemented Chang and $\mathrm{Choi}^{20)}$, was used. The modified instrument 
was used after testing for validity. The instrument was used after removing items $14 \sim 17$ with factor loadings of $<0.4$ since the cumulative covariance was $54.72 \%$. The instrument also included the following sub-items: communication, trust, affection, sensitivity, openness, and understanding. Each item was rated using a five-point Likert scale; there were a total of 14 items. A higher score denotes a higher level of interpersonal relations. In a study by Chang and $\mathrm{Choi}^{20)}$, Cronbach's $\alpha$ was 0.89 , while that in the present study was 0.846 .

\section{Data analysis}

Frequency analysis of the dental hygienists' general characteristics and job characteristics, adult attachment styles, organizational commitment, work-related stress, and interpersonal relations was performed. The independent samples t-test and one-way ANOVA were used to compare the differences in adult attachment styles, organizational commitment, work-related stress, interpersonal relations, general characteristics, and job characteristics among dental hygienists. One-way ANOVA was used to measure the differences in organizational commitment, work-related stress, and interpersonal relations among dental hygienists according to adult attachment styles. Post-hoc Scheffe test was used and showed a significance level ( $\alpha$ ) of 0.05. IBM SPSS Statistics ver. 20.0 (IBM Corp., Armonk, NY, USA) was used to analyze the data.

\section{Results}

1. Participants' adult attachment styles, organizational commitment, occupational stress, and interpersonal relations

The participants' scores according to adult attachment styles were as follows: 2.69 in the avoidance dimension and 2.65 in the anxiety dimension. Moreover, they obtained a score of 3.11 for organizational commitment, 3.40 for occupational stress, and 3.43 for interpersonal relations (Table 1).

\section{Participants' adult attachment styles}

Adult attachment styles were classified into four types based on the avoidance dimension and anxiety dimension, which were sub-items of adult attachments (Table 2). About 39 dental hygienists $(8.6 \%)$ had secure attachment, $35(7.7 \%)$ had preoccupied attachment, 162 (35.7\%) had dismissing attachment, and 218 (48.0\%) had fearful attachment. In other words, the most common adult attachment style observed in dental hygienists was fearful attachment, followed by dismissing attachment.

Table 2. Dental Hygienists' Adult Attachment Styles $(n=454)$

\begin{tabular}{lcc}
\hline \multirow{2}{*}{ Variable } & \multicolumn{2}{c}{ Anxiety attachment } \\
\cline { 2 - 3 } & Low & High \\
\hline Avoidance attachment & & \\
Low & Security & Preoccupation \\
\multirow{2}{*}{ High } & $39(8.6)$ & $35(7.7)$ \\
& Dismissing-avoidance & Fearful \\
& $162(35.7)$ & $218(48.0)$ \\
\hline
\end{tabular}

Values are presented as $\mathrm{n}(\%)$.

Table 1. Dental Hygienists' Adult Attachment Styles, Organizational Commitment, Occupational Stress, and Interpersonal Relations

\begin{tabular}{lcccccc}
\hline \multicolumn{1}{c}{ Variable } & Range & No. of items & Minimum & Maximum & Mean \pm SD & Cronbach's $\alpha$ \\
\hline Adult attachment & & & & & & \\
$\quad$ Avoidance & $1 \sim 5$ & 17 & 1.39 & 4.00 & $2.69 \pm 0.41$ & 0.775 \\
Anxiety & $1 \sim 5$ & 17 & 1.00 & 4.22 & $2.65 \pm 0.57$ & 0.857 \\
Total & $1 \sim 5$ & 34 & 1.42 & 4.00 & $2.69 \pm 0.39$ & 0.815 \\
Organizational commitment & $1 \sim 5$ & 12 & 1.00 & 5.00 & $3.11 \pm 0.73$ & 0.923 \\
Occupational stress & $1 \sim 5$ & 9 & 1.00 & 5.00 & $3.40 \pm 0.62$ & 0.842 \\
Interpersonal relations & $1 \sim 5$ & 14 & 2.00 & 5.00 & $3.43 \pm 0.43$ & 0.846 \\
\hline
\end{tabular}

SD: standard deviation. 
3. Differences in adult attachment styles, organizational commitment, occupational stress, and interpersonal relations based on the general characteristics of study participants

Based on the participants' scores on adult attachment styles by general characteristics, there were statistically significant differences in age $(\mathrm{p}=0.001)$, marital status $(\mathrm{p}<$ $0.001)$, education level $(\mathrm{p}=0.027)$, work experience $(\mathrm{p}=0.001)$, and monthly income $(\mathrm{p}=0.001)$. Post-hoc test for age showed that individuals aged $<25$ years $(2.79)$ and 25 29 years (2.74) had higher scores in items related to adult attachments than those aged $\geq 30$ years (2.63). Individuals with $<3$ years (2.80) and $3 \sim 6$ years (2.75) of work experience had higher scores in items related to adult attachments than those with $\geq 7$ years (2.64) of work experience. Individuals with monthly income of $<2,000,000$ Korean won (KRW) (2.79) had higher scores in items related to adult attachments than those with monthly income of $\geq 2,500,000 \mathrm{KRW}$ (2.64). There was a statistically significant difference in monthly income $(p=0.019)$ based on the level of organizational commitment.

With regard to levels of occupational stress, significant differences were observed in age $(p<0.001)$, marital status ( $\mathrm{p}=0.011)$, and work experience $(\mathrm{p}=0.018)$ among dental hygienists. Post-hoc test for age showed that individuals aged $<25$ years (3.62) and aged $25 \sim 29$ years (3.54) had higher scores in items related to work stress than those aged $\geq 30$ years (3.29). Individuals with $<3$ years of work experience (3.59) had higher scores than those with $\geq 7$ years (3.38) of work experience. With regard to interpersonal relations, the participants showed significant differences in age $(\mathrm{p}=0.021)$, marital status ( $p=0.003)$, education level $(\mathrm{p}<0.001)$, work experience $(\mathrm{p}=0.007)$, and monthly income $(\mathrm{p}=0.003)$. As a result of post-hoc test, individuals with $\geq 7$ years of work experience (3.50) had higher scores in items related to interpersonal relations than those with $<3$ years of work experience (3.34); moreover, individuals with monthly income of $\geq 2,500,000 \mathrm{KRW}$ had higher scores in items related to interpersonal relations than those with monthly income of $<2,000,000 \mathrm{KRW}$ (Table 3).
4. Differences in adult attachment styles, organizational commitment, occupational stress, and interpersonal relations based on the job characteristics of study participants

With regard to the scores of the participants on adult attachment styles by job characteristics, there were significant differences in job position $(\mathrm{p}=0.001)$, relationships with colleagues, $(p=0.034)$, and consideration for career change $(p=0.001)$. The results of post-hoc test showed that individuals who responded "often" (2.81), "sometimes" (2.72), and "Almost never" (2.73) to the question related to consideration for career change had higher levels of adult attachment than those who responded "never" (2.50). With regard to the organizational commitment, there were significant differences in relationships with colleagues $(p=0.001)$, experience of conflict in workplace $(\mathrm{p}<0.001)$, and consideration of career change $(\mathrm{p}=0.009)$ among dental hygienists. The results of post-hoc test showed that individuals who responded "Satisfaction" (2.99) to the question related to the relationships with colleagues had higher scores in organizational commitment than those who responded "Dissatisfaction" (2.83). In addition, individuals who responded "Almost never" (3.03) or "never" (3.05) to questions related to experience of conflict in workplace had higher scored in organizational commitment than those who responded "often" (2.86). With regard to occupational stress, there were significant differences in relationships with colleagues $(p=0.001)$, conflict in workplace $(p<0.001)$, and consideration for career change $(\mathrm{p}<0.001)$ among dental hygienists. The results of post-hoc test showed that individuals who responded "Dissatisfaction" (3.92) with their relationships with colleagues had higher levels of stress than those who responded "Normal" (3.60) and "Satisfaction" (3.44) with their relationships with colleagues. The test also showed that individuals who responded "often" (3.93) or "sometimes" (3.62) to questions related to experience with conflict in workplace had higher levels of stress than those who responded "Almost never" (3.27) or "never" (3.21). Furthermore, individuals who "often" (3.77) considered changing their careers were more likely to have higher levels of stress than those who "never" (3.04) considered 


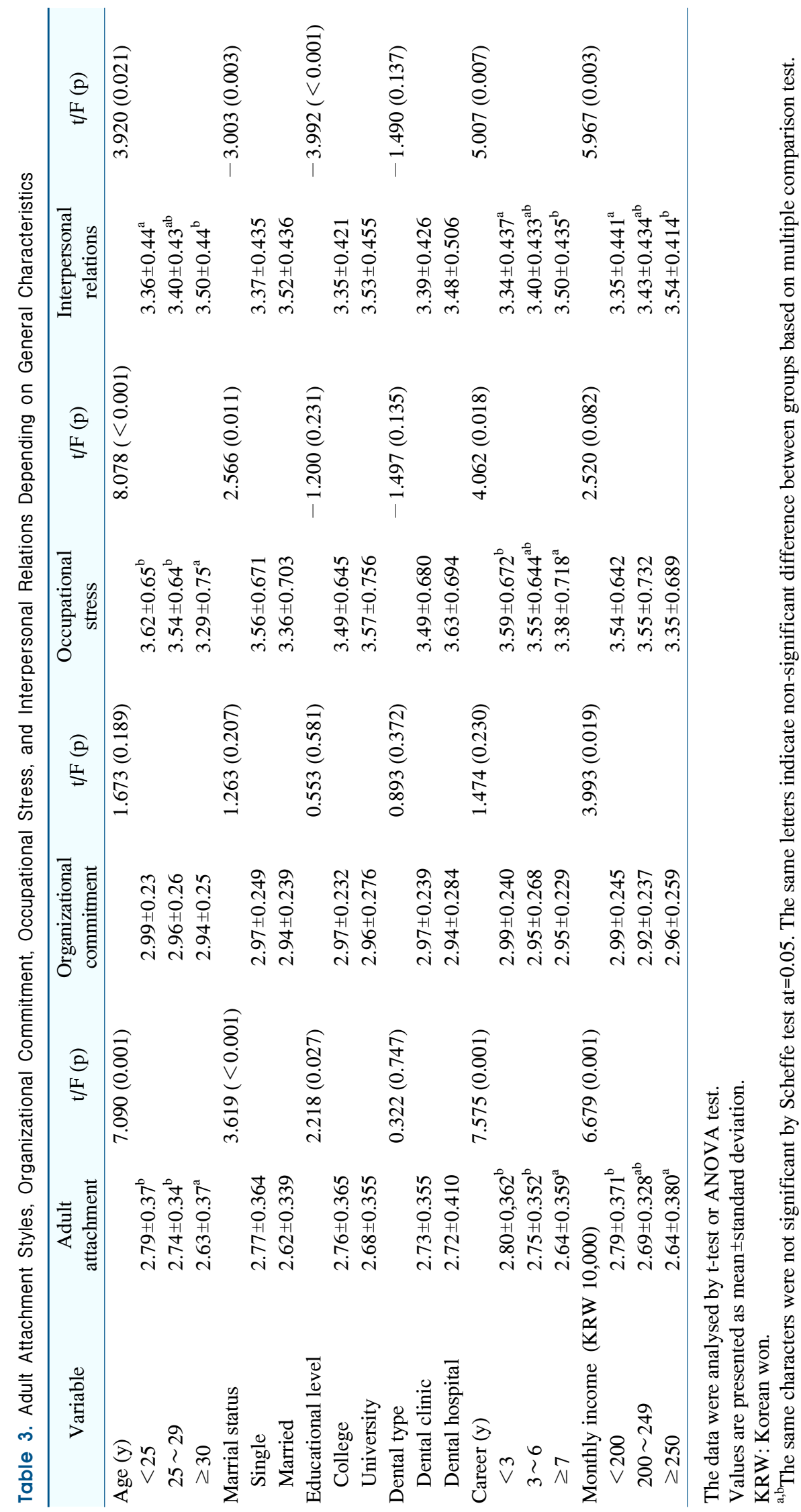




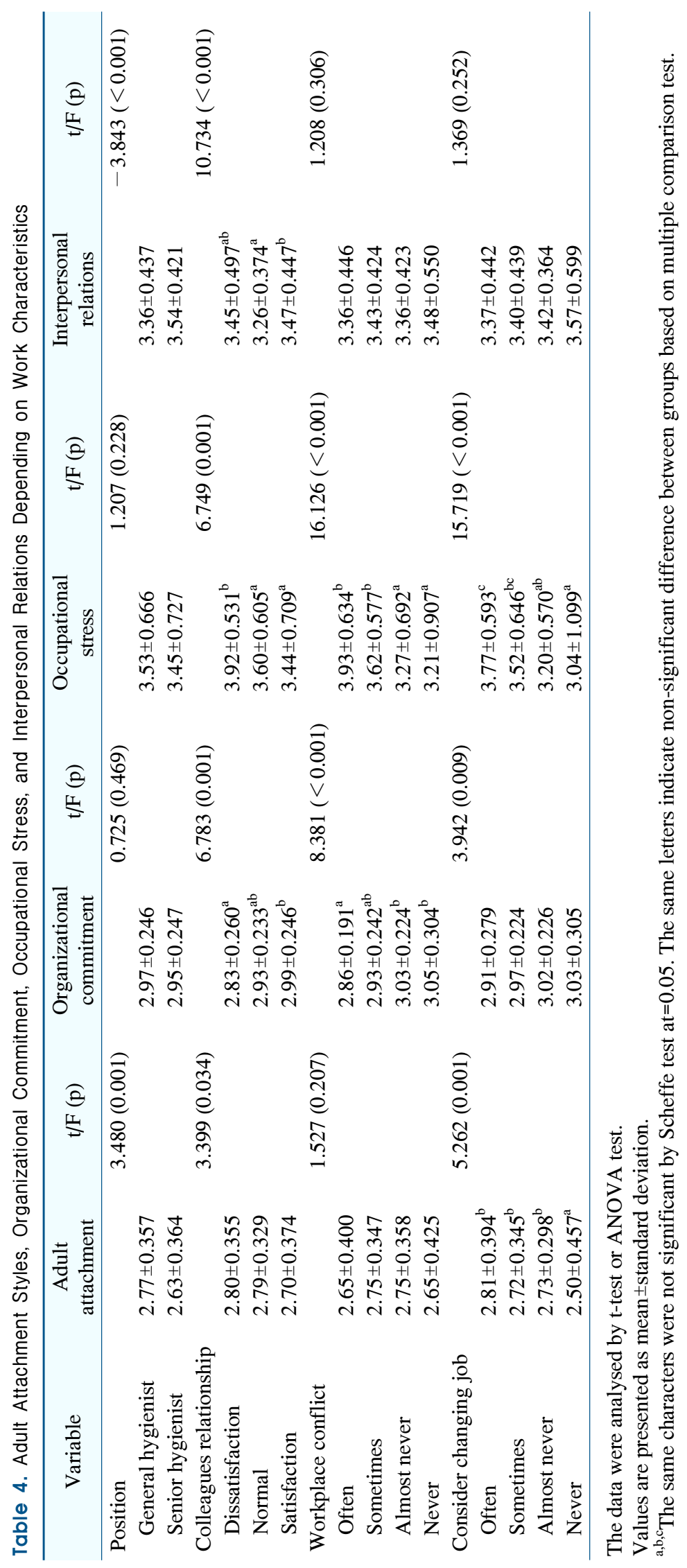


changing their careers. With regard to interpersonal relations, there were significant differences in job position $(\mathrm{p}<0.001)$ and relationships with colleagues $(\mathrm{p}<0.001)$ among dental hygienists. The post-hoc test results demonstrated that individuals who were "Satisfaction" with their relationships with colleagues had higher scores in interpersonal relations than those who responded "Normal" with their relationships with colleagues (Table 4).

\section{Differences in organizational, occupational stress,} and interpersonal relations based on the adult attachment styles

There were statistically significant differences in occupational stress and interpersonal relations by adult attachment styles $(\mathrm{p}<0.001)$. The post-hoc test results showed that individuals with preoccupied attachment (3.76) were more likely to experience work-related stress than those with dismissing attachment (3.30); moreover, individuals with secure (3.90) and preoccupied attachments (3.86) were more likely to have better interpersonal relations than those with dismissing (3.47) and fearful (3.29) attachments (Table 5).

\section{Discussion}

This study aimed to determine the adult attachment styles of clinical dental hygienists and to compare and analyze the relationships between adult attachment and organizational commitment, occupational stress, and interpersonal relations.

As no previous studies have evaluated the adult attachment styles of clinical dental hygienists, we aimed to compare our results with those of previous studies conducted among nurses. The present study included dental hygienists, and the scores of the participants in items related to adult attachment styles were as follows: 2.69 in the avoidance dimension and 2.65 in the anxiety dimension. In a study by $\mathrm{Oh}$ and Sung ${ }^{4)}$, the study participants were nurses working in university hospitals, and the scores were 2.65 in avoidance dimension and 2.80 in anxiety dimension. In a study by Jeong et al. ${ }^{21)}$, the study participants were nurses working in small or medium hospitals, and the scores were 2.57 in avoidance dimension and 2.66 in anxiety dimension. There were no significant differences in the mean scores between previous studies conducted on nurses and the present study, but the nurses working in university hospitals had higher mean scores in the anxiety dimension.

The most common adult attachment style shown in participants of the present study was fearful attachment (48.0\%) followed by dismissing attachment (35.7\%), preoccupied attachment (7.7\%), and secure attachment (8.6\%). This finding is similar to that of Oh and Sung's study ${ }^{4)}$, targeting shift nurses working in university hospitals, which reported that the most common adult attachment style was fearful attachment. On the contrary, a study by Jeong et al. ${ }^{21)}$, targeting nurses working in small or medium hospitals, and a study by $\mathrm{Oh}$ et al. ${ }^{7)}$, targeting inexperienced nurses, reported that the most common adult attachment style was secure attachment; however, their findings were in conflict with the results of the present study. These results may be related to the high level of avoidance dimension; thus, further study must be conducted to compare these results with those of general workers.

Table 5. Organizational Commitment, Occupational Stress, and Interpersonal Relations Depending on Adult Attachment Styles

\begin{tabular}{|c|c|c|c|c|c|}
\hline \multirow{2}{*}{ Variable } & \multicolumn{4}{|c|}{ Adult attachment } & \multirow{2}{*}{$\mathrm{F}(\mathrm{p})$} \\
\hline & Security & Preoccupation & Dismissing & Fearful & \\
\hline Organizational commitment & $3.23 \pm 0.942$ & $3.16 \pm 0.697$ & $3.15 \pm 0.405$ & $3.02 \pm 0.628$ & $2.391(0.068)$ \\
\hline Occupational stress & $3.50 \pm 0.719^{\mathrm{ab}}$ & $3.76 \pm 0.624^{\mathrm{b}}$ & $3.30 \pm 0.754^{\mathrm{a}}$ & $3.61 \pm 0.592^{\mathrm{ab}}$ & $8.269(<0.001)$ \\
\hline Interpersonal relations & $3.90 \pm 0.439^{\mathrm{b}}$ & $3.86 \pm 0.397^{\mathrm{b}}$ & $3.47 \pm 0.408^{\mathrm{a}}$ & $3.29 \pm 0.388^{\mathrm{a}}$ & $41.340(<0.001)$ \\
\hline
\end{tabular}

The data were analysed by ANOVA test.

Values are presented as mean \pm standard deviation.

${ }^{a, b}$ The same characters were not significant by Scheffe test at $=0.05$. The same letters indicate non-significant difference between groups based on multiple comparison test. 
By general characteristics, the adult attachment scores and occupational stress levels were low in individuals aged $<25$ years. Adult attachment levels were low, while the stress levels were high in new dental hygienists with $<3$ years of working experiences. As mentioned in a study by $\mathrm{Oh}$ et al. ${ }^{7)}$, since adult attachment is not yet fully developed, helping these individuals develop secure attachments and adapt business environment would affect the results. Hence, a separate research on new dental hygienists using adult attachment styles must be conducted. Dental hygienists with $\geq 7$ years of clinical experience with monthly income of $\geq 2,500,000 \mathrm{KRW}$ had higher levels of interpersonal relations, while new dental hygienists had higher levels of interpersonal relations in other variables. This is because experienced dental hygienists were more likely to have cumulative experience with interpersonal relations; that is, older individuals have better interpersonal skills ${ }^{22)}$.

With regard to adult attachment scores by job characteristics of dental hygienists, those without job position (2.77), who were not satisfied with their relationships with colleagues (2.80), or who often considered a career change (2.81) had lower scores on adult attachments. This finding may suggest that if the level of attachment is low, one might be unable to develop a more fulfilling relationship with colleagues due to sensitivity to stress and fear of engaging into interpersonal relations; moreover, an individual will more likely to consider changing his or her career to avoid those situations. The scores in organizational commitment were high when there was satisfaction with peer relationships $(p=0.001)$ and absence of conflict in workplace $(p<0.001)$. Contrary to this, occupational stress level was higher when there was dissatisfaction with peer relationships $(p=0.001)$, conflict in workplace $(\mathrm{p}<0.001)$, and frequent consideration for a career change $(p<0.001)$. As the occupational stress was higher, individuals had higher intension for career change $^{23)}$, and the dental hygienists who had a plan to change their career were influenced by occupational stress $^{24)}$. Therefore, positive emotions are needed to satisfy the working environment, and it is necessary to conduct a study that specifically determines the factors that can reduce the stress level rather than simply identifying the stress level.

In terms of organizational commitment, occupational stress, and interpersonal relations by adult attachment style presented in this study, the secure attachment style had the highest score while fearful attachment style had the lowest score in the organizational commitment. Organizational commitment refers to identification of oneself with an affiliated organization and active participation in the organization ${ }^{21)}$. Previous studies showed similar results demonstrating that individuals with secure attachment have a higher level of commitment to the job than those with insecure attachment ${ }^{4,25)}$. Individuals with preoccupied attachment had the highest score on occupational stress, followed by individuals with fearful attachment. The result was somewhat contrary to studies by $\mathrm{Oh}$ and Sung ${ }^{4)}$ and Jeong et al. ${ }^{21)}$, which showed that individuals with fearful attachment had the highest level of occupational stress. Preoccupied attachment styles are characterized by a desire for intimate relationships with others and a fear that others would hate them ${ }^{1)}$. The desire and fears of forming relationships with various people, such as colleagues and patients, may have caused the stress and were probably influenced by one's tendency to develop a fearful attachment, which is an insecure attachment with high avoidance and anxiety.

The study results showed that the highest styles in organizational commitment and occupational stress are different. Therefore, occupational stress level can be influenced by the nature of adult attachment styles. Individuals with the secure attachment style showed better interpersonal relations than those with other attachment styles. Individuals with dismissing attachment style had low occupational stress levels, followed by individuals with the secure attachment style. An adult with a secure attachment did not experience difficulties in forming an intimate relationship and was not afraid of being with others ${ }^{1)}$. Participants in this study reported high levels of satisfaction with interpersonal relations, which was also similar to that reported in previous studies ${ }^{7)}$. Individuals with fearful attachment, which is characterized by high avoidance, high anxiety scores, and characteristics of fear and difficulty with interpersonal relations, garnered the lowest score for interpersonal relations. Individuals with 
dismissing style desire to be independent with their relationship with other people ${ }^{1}$. In the present study, the low levels of occupational stress and interpersonal relations seemed to be the result of low interest and activeness in interpersonal relations.

The results showed that there was a significant difference between the adult attachment styles of dental hygienists with high levels of organizational commitment and interpersonal relations - positively affecting the work in the dental hospitals/clinics - and those with adult attachment styles associated with high occupational stress - which had a negative effect. Most of the participants in the present study appeared to have fearful attachment style; thus, future studies using dental hygienists alone who have fearful attachment style are warranted.

The result of this study is not applicable to all clinical dental hygienists as the samples were selected using convenience sampling; as only basic statistics were conducted in this study, some limitations were identified. However, the present study not only emphasizes the importance of improving the working environment while respecting the individuals' tendency, but it also suggests the importance of forming desirable secure attachment by identifying the specific type of dental hygienists and the necessity of developing such attachment. When allocating dental hygienists or conducting employee training, it is important to identify the individual type first and implement an appropriate education program before providing a comprehensive education. Therefore, it is necessary to develop and apply a personal attachmentenhancing program that can enhance the adult attachment style of clinical dental hygienists and institutional support for differentiated clinical education should be provided.

\section{Notes}

\section{Conflict of interest}

No potential conflict of interest relevant to this article was reported.

\section{Ethical approval}

The study was approved by the Public Institutional Review Board of Ministry of Health and Welfare (IRB
No. P01-201808-23-001).

\section{ORCID}

Mi-Hyun Choi, https://orcid.org/0000-0001-9824-4764

Hee-Hong Min, https://orcid.org/0000-0002-6587-5639

\section{References}

1. Jung OB, Jung SH, Hwang HJ: Attachment and development. Hakjisa, Seoul, pp.15-32, 2009.

2. Bartholomew K, Horowitz LM: Attachment styles among young adults: a test of a four-category model. J Pers Soc Psychol 61: 226-244, 1991. https://doi.org/10.1037/0022-3514.61.2.226

3. Brennan KA, Clark CL, Shaver PR: Self-report measurement of adult attachment: an integrative overview. In: Shimpson JA, Rholes WA, eds. Attachment theory and close relationships. Guilford Press, New York, pp.46-76, 1998.

4. Oh EJ, Sung KM: Nurses' organizational commitment, job satisfaction and job stress according to their adult attachment styles. J East-West Nurs Res 17: 117-124, 2011.

5. Kim GE: Personality characteristics and stress coping strategies related with adult attachment styles and factors. Korean J Couns Psychother 16: 53-69, 2004.

6. Park JH, Jung SK: Depression, anger expression and professional self-concept according to adult attachment style in university nursing. Asia-Pac J Multimed Serv Converg Art Humanit Sociol 7: 519-532, 2017.

7. Oh EJ, Lee SY, Sung KM: Interpersonal relations, hope, professional self-concept and turnover intention according to adult attachment styles in early stage nurses. J Korean Acad Nurs Adm 19: 491-500, 2013. https://doi.org/10.11111/jkana.2013.19.4.491

8. Moon SJ, Ka KH, Ku IY, Kim EH: The research on job stress, self-efficacy, and organizational commitment by the job characteristics of dental hygienists in rural areas. J Korean Isl 29: 147-170, 2017.

9. Choi JH, Choi JS: Effect of mediating variable on the relationship between job stress and stress response among clinical dental hygienists. J Dent Hyg Sci 14: 114-122, 2014.

10. Yoon SU, Nam IS: Relations between emotional labor and sleep health according to the personality types of dental hygienists. J Dent Hyg Sci 11: 243-249, 2011. 
11. Kwon SJ, Bae SK: Analysis of impact factors of affective commitment in organization of dental hygienist in Busan areafocused on quality of working life(QWL)-. J Korea Contents Assoc 9: 156-165, 2009.

https://doi.org/10.5392/jkca.2009.9.5.156

12. Yoon SW, Hong YH: The relationship between job stress and social-psychosocial stress among dental-workers in some area. J Korean Acad Dent Hyg 12: 137-149, 2010.

13. Kim JH, Kim MY: The study of DISC behavioral patterns on job satisfaction, organization commitment and job stress in nurses and dental hygienist. Korean J Health Serv Manag 6: 73-82, 2012. https://doi.org/10.12811/kshsm.2012.6.4.073

14. Hwang KY: The relationship between attachment style, identity and interpersonal relation tendency of university students. Unpublished master's thesis, Wonkwang University, Iksan, 2001.

15. Allen NJ, Meyer JP: The measurement and antecedents of affective, continuance and normative commitment to the organization. J Occup Psychol 63: 1-18, 1990. https://doi.org/10.1111/j.2044-8325.1990.tb00506.x

16. Oh EJ: A study on nurses' organizational commitment, job satisfaction and job stress according to their adult attachment styles. Unpublished master's thesis, Gyeongsang University, Jinju, 2011.

17. Kim MJ, Gu MO: The development of the stress measurement tool for staff nurses working in the hospital. $\mathrm{J}$ Nurs Acad Soc 14: 28-37, 1984.

https://doi.org/10.4040/jnas.1984.14.2.28
18. Guerney BG: Relationship enhancement: skill-training programs for therapy, problem prevention, and enrichment. Jossey-Bass Publishers, San Francisco, pp.381-391, 1977.

19. Moon SM: A study on the effect of human relations training of university students. J Gyeongsang Natl Univ 19: 195-204, 1980.

20. Chang HS, Choi BY: The effect of adolescent's background, family self-esteem, interpersonal relations disposition on etiquette implementation. J Korean Fam Resour Manag Assoc 13: 23-41, 2009.

21. Jeong ES, Kim JW, Bae SY: Job stress and organizational commitment with regard to adult attachment style of small and medium-sized hospital nurses. Korea J Hosp Manag 23: 72-86, 2018.

22. Han JH, Kim KE: Interpersonal skills caused by emotional intelligence and ego resilience of students majoring in dental hygiene. J Dent Hyg Sci 15: 746-752, 2015. https://doi.org/10.17135/jdhs.2015.15.6.746

23. Kim YK, Choi MS: The influence of job stress of male and female dental hygienists on turnover intention. J Korean Acad Dent Hyg 19: 67-78, 2017.

24. Lee ML, Lee HC: The effect of job stress and turnover intention in dental hygienists. J Korea Entertain Ind Assoc 11: 249-258, 2017. https://doi.org/10.21184/jkeia.2017.04.11.3.249

25. Roh KH, Choi HK: The influence of attachment and love on organizational activity commitment. J Fam Relat 13: 77-112, 2008. 